\title{
On the Hyperferritinemia and Hereditary Cataract Syndrome
}

\author{
Maria José Pérez-Lucena ${ }^{1}$, María Sierra Moreno-Rosel ${ }^{2}$, María Sagarra-Tió ${ }^{2}$ Jordi Félez $^{2 *}$ \\ ${ }^{1}$ Area Bàsica de Salut (ABS) Serraparera, Cerdanyola del Vallès, Barcelona, Spain; ${ }^{2}$ Area Bàsica de Salut (ABS) Canaletes, Institut \\ Català de la Salut (ICS), Cerdanyola del Vallès, Barcelona, Spain. \\ Email: *jordifelez@gmail.com
}

Received December $11^{\text {th }}, 2011$; revised January $26^{\text {th }}$, 2012; accepted February $10^{\text {th }}, 2012$

\begin{abstract}
Introduction: Mutations in the promoter region of ferritin light gene can induce an uncontrolled over expression of this protein. Consequently, ferritin is found in serum at very high levels $(\sim 1000 \mathrm{ng} / \mathrm{mL})$ and it accumulates in the crystalline lens, generating cataracts. This entity is known as hyperferritin and hereditary cataract syndrome (HHCS) which is inherited in an autosomal dominant manner. Case Presentation: We describe a family affected by HHCS. The proband was identified among subjects submitted to a biological screening for hemochromatosis. He had very high levels of serum ferritin ( $\sim 900 \mathrm{ng} / \mathrm{mL}$ ) with normal transferrin saturation (TS). The proband has a single H63D HFE-gene mutation and normal HAMP-gene. He was submitted to periodical phlebotomies that induced anemia and a decrease in TS but no changes on serum ferritin levels. Analyses of promoter region of ferritin-light chain gene showed a 39C > T mutation, responsible for HHCS. The proband's sister carried also this mutation. Both subjects had developed cataracts. Discussion: Similar to the first family described carrying this syndrome and to other cases reported, the proband was erroneously submitted to phlebotomies. Clinical consequences are illustrated in this report. HHCS is an infrequent entity which has to be correctly identified. The unique therapeutic approach to this syndrome must be cataract surgery.
\end{abstract}

Keywords: Hyperferritinemia; Iron Overload; Cataract; Hyperferritinemia and Hereditary Cataract Syndrome (HHCS)

\section{Introduction}

Prevalence of congenital cataract is estimated in 2.2 - 2.4 over 10,000 births [1]. Approximately $30 \%$ of patients suffering cataract have familiar history and frequently they are transmitted in an autosomal dominant manner [1]. The hyperferritinemia and hereditary cataract syndrome (HHCS) has a relevant role among these syndromes. HHCS was first described in 1995 and it is characterized by an early onset of cataracts and the detection of very high serum ferritin levels [2-4]. It has an ubicous geographic distribution, affecting individuals from Europe, America, Australia and India [5]. This syndrome has a unique clinical complication: the presence of cataracts with distinct morphology and variable period of onset which depends on both the molecular type of HHCS and on the ethnicity of affected individuals [2-4]. HHCS patients have high serum ferritin levels but without iron-overload (reviewed in [6]). The association of cataract with hyperferritinemia and the fact that measurement of serum ferritin levels is broadly used in routine blood tests, confers an emergent relevance to HHCS which should be identified by primary care practi-

${ }^{*}$ Corresponding author. tioners, ophthalmologists, pediatricians, gastroenterologists and hematologists. We hereby describe a patient affected by HHCS that was detected during a multicenter primary care case-finding study on hereditary hemochromatosis carried out by our group among 2739 individuals [7].

\section{Case Report}

A 24 year-old male was enrolled in a biological study for screening of hemochromatosis. In a first blood test, hemoglobin of $15.8 \mathrm{~g} / \mathrm{L}$ and serum ferritin levels of 900 $\mathrm{ng} / \mathrm{mL}$ were detected as unique abnormalities. Transferrin saturation (TS) was normal. Hyperferritinemia was confirmed in two additional measurements. The proband has a single H63D HFE-gene mutation and normal HAMP-gene. Panel A of Figure 1 depicts iron-related abnormalities found in his family. Due to elevated serum ferritin levels found, the patient was submitted to six 400 $\mathrm{mL}$ phlebotomy that induced anemia and a decrease in TS but without changes on serum ferritin levels (Panels $\mathrm{B}$ and C). Medical history was reassessed and then, a cataract surgery was identified at early childhood. Analysis of ferritin light chain (FtL) promoter showed a 39C > 


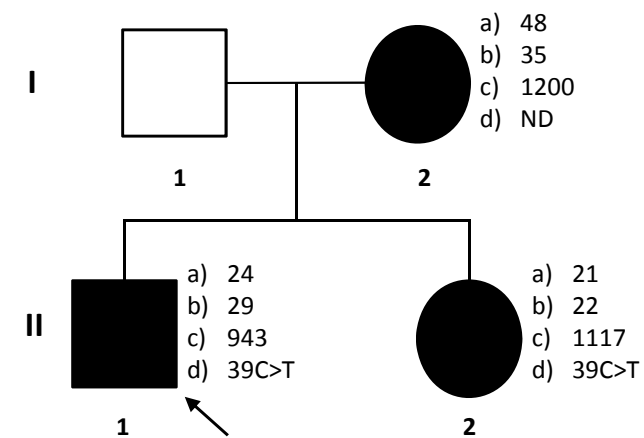

(A)

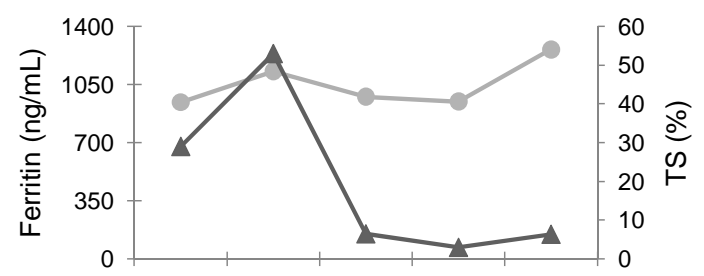

(B)

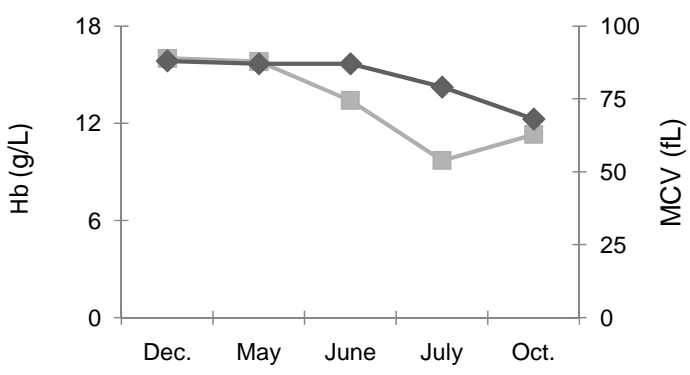

(C)

Figure 1. Family affected by Hyperferritinemia and Hereditary Cataract Syndorme (HHCS). Panel A: Circles, women. Square, men. Affected members filled in black. Propositus indicated by arrow. a) (in small letter) age; b) (in small letter) Transferrin Saturation (\%); c) (in small letter) serum ferritin levels (ng/mL); d) (in small letter) Type of mutation in the promoter region of light chain ferritin gene; ND) Not determined. There are no data on the propositus's father. Panel B: Changes in serum ferritin levels (circles) and transferrin saturation (TS, triangles) induced by phlebotomy (since month of May). There is a fast decrease in TS without marked changes in serum ferritin levels that remain 900 ng/mL. Panel C: Hemoglobin (g/mL, diamonds) and MCV (fL, squares) changes induced by phlebotomy (for details, see the text).

$\mathrm{T}$ mutation, responsible for HHCS. This mutation was also indentified in the proband's sister (21 year-old) and not determined in mother's proband (48 year-old). Both were also submitted to crystalline surgery at early childhood and had normal transferrin saturation measurements but very high ferritin levels (>1000 ng/mL, see details in figure).

\section{Discussion}

Ferritin is a multi polymer formed by two molecules of different molecular weight. Whereas heavy chain ferritin gene is regulated at a transcriptional level by several oncogenes, cytokines, etc., FtL gene is mainly modulated by iron at a translation level by a number of Iron Regulatory Proteins (IRP's 1 - 2) [8]. Abnormal up-regulation of FtL gene may be due to up to 31 distinct base mutations of these regions which induce very high serum ferritin levels $[9,10]$. In these cases, ferritin accumulates in lymphocytes, without clinical consequences and in crystalline lens developing cataracts [5]. The high ferritin levels is consequence of the increased synthesis of the FtL chain but not of total body iron, since the light chain ferritin does not participate in iron oxidation and storage (reviewed in 6). Mutations in FtL are extremely rare and associated to either HHCS or to a novel genetic dominant unexplained hyperferritinemia that has been recently described in a large family $(n=22)$ [11]. In these subjects, mutations in IRE sequence of FtL promoter were not identified but the authors found a single mutation (p.Thr30Ile) in the coding sequence FtL in half the familial and in few isolated cases but not in more than 500 normal controls. This new unexplained hyperferritinemia is not associated to relevant clinical data.

We have reported a new Spanish family affected by HHCS due to a 39C > T substitution within the IRE region of FtL promoter. The proband of this family carried also a single H63D mutation of HFE gene, fact also found in other recently described families in Spain [12]. In HHCS, hyperferritinemia is not paralleled with a proper iron-overload and thus no pathologies associated to iron excess are developed. Thus, no interventions such as phlebotomy are needed in these patients. However, some affected subjects, such as the one described in this letter and others previously reported [2,6,9], have been treated or submitted to biopsy to assess liver iron-overload. Sometimes, these patients are also stigmatized as carriers of an iron-overload syndrome. These practices are due to the unfamiliarity of the HHCS. Thus, we think it is important to give diffusion to new cases of HHCS and to reinforce a correct therapeutic approach of this syndrome.

\section{Acknowledgements}

We thank Dr. Laura Cremonesi, Unit of Genomics for the Diagnosis of Human Pathologies; DIBIT2; San Raffaele Scientific Institute: Milan, Italia, for molecular characterization of the HHCS described. This work was supported by FIS 03/0459 and FIS 08/0956.

\section{REFERENCES}

[1] J. S. Rahi, "Childhood Blindness: A UK Epidemiological Perspective,” Eye (Lond), Vol. 21, 2007, pp. 1249-1253. doi:10.1038/sj.eye.6702837 
[2] D. Girelli, R. Corrocher, D. Bisceglia, et al., "Molecular Basis for the Recently Described Hereditary Hyperferritinemia-Cataract Syndrome: A Mutation in the IronResponsive Element of Ferritin L-Subunit Gene (the 'Verona Mutation')," Blood, Vol. 86, 1995, pp. 40504053.

[3] C. Beaumont, P. Leneuve, I. Devaux, et al., "Mutation in the Iron Responsive Element of the L Ferritin mRNA in a Family with Dominant Hyperferritinaemia and Cataract," Nature Genetics, Vol. 11, 1995, pp. 444-446. doi:10.1038/ng1295-444

[4] D. Bonneau, I. Winter-Fuseau, M. N. Loiseau, et al., "Bilateral Cataract and High Serum Ferritin: A New Dominant Genetic Disorder?” Journal of Medical Genetics, Vol. 32, No. 10, 1995, pp. 778-779. doi:10.1136/jmg.32.10.778

[5] V. Vanita, J. F. Hejtmancik, H. C. Hennies, et al., "Sutural Cataract Associated with a Mutation in the Ferritin Light Chain Gene (FTL) in a Family of Indian Origin,” Molecular Vision, Vol. 21, 2006, pp. 93-99.

[6] C. Camaschella and E. Poggiali, "Towards Explaining 'Unexplained Hyperferritinemia',' Haematologica, Vol. 94, No. 3, 2009, pp. 307-309. doi:10.3324/haematol.2008.005405

[7] N. Freixenet, M. S. Moreno-Rosel, M. J. Barceló, et al., "Detection of Hereditary Hemochromatosis and Biochemical Iron-Overload in Primary Care: A Multicenter Case-Finding Study in Spain,” American Journal of He- matology, Vol. 85, No. 4, 2010, pp. 294-296. doi:10.1002/ajh.21634

[8] K. J. Hintze and E. C. Theil, "Cellular Regulation and Molecular Interactions of the Ferritins," Cellular and Molecular Life Sciences, Vol. 63, No. 5, 2006, pp. 591600. doi:10.1007/s00018-005-5285-y

[9] M. Cazzola, G. Bergamaschi, L. Tonon, et al. "Hereditary Hyperferritinemia-Cataract Syndrome: Relationship between Phenotypes and Specific Mutations in the IronResponsive Element of Ferritin Light-Chain mRNA," Blood, Vol. 90, No. 2, 1997, pp. 814-821.

[10] G. Milloning, M. U. Muckenthaler and S. Mueller, "Hyperferritinaemia-Cataract Syndrome: Worldwide Mutations and Phenotype of an Increasingly Diagnosed Genetic Disorder,” Human Genomics, Vol. 4, No. 4, 2010, pp. 250-262.

[11] C. Kannengiesser, A. M. Jouanolle, G. Hetter, et al., “A New Missense Mutation in the L Ferritin Coding Sequence Associated with Elevated Levels of Glyscosylated Ferritin in Serum and Absence of Iron Overload," Haematologica, Vol. 94, No. 3, 2009, pp. 335-339. doi:10.3324/haematol.2008.000125

[12] J. A. García Erce, T. Cortés, L. Cremonesi, et al., "Hiperferritinemia Familiar y Cataratas Congenitas Asociadas a Mutación del Gen HFE. Dos Nuevas Familias Españolas y una Nueva Mutación (A37T: 'Zaragoza'),” Medicina Clínica (Barc), Vol. 127, No. 2, 2006, pp. 5558. 